Mens

Revue d'histoire intellectuelle et culturelle

mens

\title{
À l'assaut de Montréal : les chanteurs country-western dans les studios de la métropole, 1942-1958
}

\section{Catherine Lefrançois}

Volume 16, numéro 1, automne 2015

Vie musicale amateur, populaire et américaine à Montréal, 1918-1958

URI : https://id.erudit.org/iderudit/1038985ar

DOI : https://doi.org/10.7202/1038985ar

Aller au sommaire du numéro

Éditeur(s)

Centre de recherche en civilisation canadienne-française

ISSN

1492-8647 (imprimé)

1927-9299 (numérique)

Découvrir la revue

Citer cet article

Lefrançois, C. (2015). À l'assaut de Montréal : les chanteurs country-western dans les studios de la métropole, 1942-1958. Mens, 16(1), 107-138.

https://doi.org/10.7202/1038985ar

\section{Résumé de l'article}

L'imaginaire campagnard associé à la chanson country-western masque une réalité méconnue. Entre 1942, année du premier enregistrement sur disque de Roland Lebrun, et 1958, année de la fondation de la première maison de disques indépendante spécialisée dans la production d'enregistrements country-western, le genre se structure en partie autour de pratiques artistiques et de réseaux urbains. Cet article propose une histoire culturelle de la chanson country-western par l'intermédiaire, notamment, de ses pratiques médiatiques. Au premier plan de cette analyse se trouve la radio, qui, en faisant une place inédite aux amateurs, permet aux premiers chanteurs country-western non seulement de se faire connaître et de se professionnaliser, mais aussi de développer une relation de grande proximité avec le public. Un bref portrait du rôle du studio d'enregistrement et des maisons de disques, qui offrent un revenu stable à ces artistes, révèle un genre largement apprécié. D’un studio à l'autre, Montréal, aux côtés de villes industrielles importantes (Drummondville et Trois-Rivières, par exemple), joue un rôle prédominant. 


\section{À l'assaut de Montréal : les chanteurs country-western dans les studios de la métropole, 1942-1958}

Catherine Lefrançois

Université Laval

\section{Résumé}

L'imaginaire campagnard associé à la chanson country-western masque une réalité méconnue. Entre 1942, année du premier enregistrement sur disque de Roland Lebrun, et 1958, année de la fondation de la première maison de disques indépendante spécialisée dans la production d'enregistrements country-western, le genre se structure en partie autour de pratiques artistiques et de réseaux urbains. Cet article propose une histoire culturelle de la chanson country-western par l'intermédiaire, notamment, de ses pratiques médiatiques. Au premier plan de cette analyse se trouve la radio, qui, en faisant une place inédite aux amateurs, permet aux premiers chanteurs country-western non seulement de se faire connaître et de se professionnaliser, mais aussi de développer une relation de grande proximité avec le public. Un bref portrait du rôle du studio d'enregistrement et des maisons de disques, qui offrent un revenu stable à ces artistes, révèle un genre largement apprécié. D'un studio à l'autre, Montréal, aux côtés de villes industrielles importantes (Drummondville et Trois-Rivières, par exemple), joue un rôle prédominant. 


\section{Abstract}

This paper examines a few aspects of the cultural history of countrywestern music in Quebec. The rural imagery of the songs, with their cowboys, their farms, their mountains, hides an important phenomenon; at its beginnings, the genre heavily relies on urban networks and urban cultural practices. This is especially important during the structuration of the genre, between 1942, when Roland Lebrun records his first songs, and 1958, when the genre finally has its own independent record companies. During these 16 years, among other strategies, country-western artists use the media to start and promote their careers. Here, the radio plays an important role; with its openness to amateurs, it allows these artists to become professionals and to develop an intimate relationship with their audience. Recording studios provide them with a regular income between touring seasons. From one studio to another, Montreal, as well as important industrial cities of the time (Drummondville, Trois-Rivieres), is an important protagonist in the structuration and the dissemination of the new genre, which deeply relies on urbanity and modernity despite the traditional image it tries to project at the same time.

Dans son important ouvrage Creating Country Music: Fabricating Authenticity, le sociologue Richard Peterson retrace l'histoire culturelle, industrielle et sociale de la musique country aux ÉtatsUnis. Il y explique, entre autres, comment la mise en valeur de la ruralité dans la musique country, qui culmine avec la cristallisation de l'authenticité comme valeur centrale du genre ${ }^{1}$, survient dans un contexte où ceux qui la produisent et qui l'écoutent se sont fortement

\footnotetext{
1 Selon Peterson, l'authenticité de la musique country s'est lentement constituée autour de représentations et de codes bien précis. Il fait remonter le processus aux tout premiers débuts du genre, au milieu des années 1920, et considère que celui-ci s'achève définitivement en 1953, avec la mort de Hank Williams, alors devenu l'icône du country authentique (Richard Peterson, Creating Country Music: Fabricating Authenticity, Chicago, University of Chicago Press, 1997, p. 81-94).
} 
urbanisés ${ }^{2}$. Constituant dès lors davantage l'expression d'une certaine nostalgie que le témoignage d'un mode de vie, elle révèle une ruralité rêvée, imaginée, fortement idéalisée. Au Québec, les premiers chanteurs country-western font leurs débuts pendant les années 1940; leurs parcours individuels et leur inscription dans le monde médiatique sont alors le reflet d'une société fortement industrialisée et urbanisée.

Roland " le soldat " Lebrun amorce sa carrière à la radio et sur disque en 1942 avec ses chansons sentimentales et ses chansons sur la guerre. Auteur-compositeur-interprète amateur, Lebrun s'accompagne à la guitare et chante d'une voix nasale, deux caractéristiques qui évoquent pour le public de l'époque les cow-boys chantants d'Hollywood et la musique country diffusée sur les ondes américaines ${ }^{3}$. Le succès de Lebrun pousse les maisons de disques à rechercher des artistes reprenant la même formule; Paul Brunelle enregistre son premier disque en 1944, suivi par Willie Lamothe en 1946 puis par Marcel Martel en 1947. Bien que de nombreux autres chanteurs country-western soient actifs au cours de la décennie, ces trois artistes sont ceux qui auront la carrière la plus durable et qui enregistreront le plus de disques dans les années 1940 et 1950. Les successeurs de Roland Lebrun adoptent résolument l'image du cow-boy : ils chantent non seulement l'Ouest, les montagnes, les prairies et les chevaux, mais aussi les campagnes et les régions du Québec, l'amour et l'enfance. Ils adoptent plusieurs techniques vocales typiques de la

2 Ibid., p. 185.

3 Les chansons enregistrées par Roland Lebrun chez Starr entre 1942 et 1945 traitent avant tout de la guerre, de romance et d'amour filial. Ce n'est qu'en 1946, à la suite du grand succès de Paul Brunelle et de Willie Lamothe, qu'il enregistre deux chansons portant sur des thèmes explicitement rattachés à l'univers western, telles La vie d'un cow-boy [1946] et Mort d'un cow-boy des prairies [1947]. Les dates placées entre crochets sont présumées correspondre à la parution ou à l'enregistrement. Celles placées entre parenthèses sont confirmées. Toutes les dates relatives aux enregistrements sonores mentionnés ont été établies par l'historien Robert Thérien et paraissent dans des notes de livrets qu'il a signées, qui accompagnent diverses rééditions numériques de ces enregistrements historiques. 
voix country, telles la nasalisation, la cassure vocale, les sanglots stylisés et l'alternance entre le premier et le second mode de phonation ${ }^{4}$, et ils mettent à leur répertoire quelques adaptations en français de succès country.

Cet article propose une histoire culturelle de la chanson countrywestern au Québec et insistera sur les divers facteurs qui ont permis la circulation de ce genre musical et le changement de statut professionnel de ses représentants, des musiciens et des chanteurs amateurs qui sont devenus des artistes du disque et de la radio. Il sera plus précisément question de la période de structuration du genre, qui s'amorce avec les débuts sur disque de Roland Lebrun en 1942 et qui se termine avec la création de la première maison de disques indépendante à produire des artistes country-western en $1958^{5}$. Entre ces deux dates, le modèle proposé par Lebrun, celui de l'auteurcompositeur-interprète s'accompagnant à la guitare dans un style country, fera de nombreux émules. Nous porterons une attention particulière à la radio. En effet, elle a joué un rôle prépondérant dans la professionnalisation des chanteurs country-western, qui l'investissent de plusieurs manières, et dans le développement d'une relation de grande proximité avec le public, qui sera, vingt ans plus tard, au cœur de l'authenticité qui caractérise le country-western. On le verra, l'histoire médiatique du country-western en révèle le caractère en partie urbain et largement moderne. Elle met également en lumière

\footnotetext{
${ }^{4}$ Pour des analyses de la voix country-western, consulter Catherine Lefrançois, " "Le train qui siffle" : nostalgie et modernité dans la chanson country-western au Québec ", Volume! La revue des musiques populaires, vol. 11, nº 1 (2014), p. 69-81; et Catherine Lefrançois, "La nasalisation dans la chanson country-western : un cas de phonostylistique ", La Revue musicale OICRM, vol. 1, n 1 (novembre 2012), [En ligne], [www.revuemusicaleoicrm.org].

5 Entre 1942 et 1958, en effet, les chanteurs country-western enregistrent pour des compagnies de disques généralistes, et le genre ne possède aucune institution autonome. La structuration s'opère progressivement pour se conclure - après à la crise économique de la fin des années 1970, la crise du disque qui lui succède et la disparition de la plupart des compagnies de disque country-western - par un repli vers l'autoproduction, l'autopromotion et la constitution de canaux de diffusion distincts.
} 
son succès populaire important, à l'opposé de l'image habituelle de musique marginale.

\section{Ruralité ou urbanité?}

Bien qu'ils incarnent des cow-boys et qu'ils chantent essentiellement la campagne, ces artistes sont issus de familles ouvrières et, en parallèle avec leurs activités musicales, ils occupent tous, à leurs débuts, des métiers industriels dans des villes régionales importantes. Si les familles de ces chanteurs peuvent être d'origine urbaine récente, peu d'artistes country-western pour lesquels nous possédons des informations biographiques ont pratiqué un métier agricole, à l'exception peut-être de Paul Brunelle ${ }^{6}$. Les pionniers du countrywestern sont principalement des travailleurs d'usine non qualifiés dont le parcours s'inscrit dans le mouvement d'urbanisation que connaissent plusieurs régions du Québec après la Crise. Roland Lebrun était le fils d'un ouvrier du moulin à scie de Saint-Léon-leGrand, dans la vallée de la Matapédia. Il a lui-même quitté la Gaspésie pour s'engager à Shawinigan dans une usine de pâtes et papiers. Démobilisé, il reprend un emploi en dehors du milieu

${ }^{6}$ Pour un article publié dans Le magazine Maclean en 1965, Gérald Godin a rencontré Willie Lamothe, Marcel Martel et Paul Brunelle. Ce dernier y est présenté comme un "paysan ". Le chanteur raconte en effet que son père était cultivateur et qu'il est donc "le seul vrai habitant des trois " (Gérald Godin, "Ils ont inventé le cowboy québécois ", Le magazine Maclean, vol. 5, nº 12 (décembre 1965), p. 40). Un dossier sur la chanson en Montérégie, préparé par Mario Gendron à partir d'archives, précise, au contraire, que Paul Brunelle est né dans une famille ouvrière (Mario Gendron, "Paul Brunelle ", Portraits de la culture montérégienne: la chanson western, sur le site Chanson d'expression française, [http://www.archivessh. qc.ca/culture/frameset_chanson.html] [23 mai 2016]). Peut-être le père de Paul Brunelle a-t-il exercé les deux types de métiers ou encore un métier agricole non spécialisé, ce qui était courant à l'époque. Le père de Marcel Martel a occupé plusieurs emplois dans le secteur ouvrier et a tenté sa chance comme agriculteur en faisant l'acquisition d'une terre (Marcel Martel et André Boulanger, Au jardin de mes souvenirs, Drummondville, Éditions de Mortagne, 1983, p. 19-23), et celui de Willie Lamothe a été aide-cultivateur sur plusieurs fermes (Diane Le Serge, Willie Lamothe : trente ans de show-business, Montréal, Publioption, 1975, p. 15). 
artistique, qu'il devra conserver toute sa vie ${ }^{7}$. Willie Lamothe a été ouvrier dans une " cannerie " ${ }^{8}$ puis à la manufacture Goodyear, deux entreprises situées à Saint-Hyacinthe, jusqu'à ce qu'il puisse vivre des tournées et des redevances 9 . Son père, Eugène Lamothe, qui était journalier et travailleur pour une compagnie de chemin de $\mathrm{fer}^{10}$, a également exercé le métier de tanneur ${ }^{11}$. Paul Brunelle aurait travaillé à l'usine Miner Rubber de Granby ${ }^{12}$. Quant à Marcel Martel, il a occupé de nombreux emplois en tant que manœuvre et ouvrier. À quinze ans, il travaille à l'usine Dominion Silk de Drummondville $^{13}$, monte dans les chantiers à l'hiver 1945-1946 ${ }^{14}$, devient livreur de lait pour la laiterie Leclerc ${ }^{15}$, à Granby, puis, de retour à Drummondville, il travaille dans une cour à bois. En 1960, il déménage avec sa famille aux États-Unis pour tenter de guérir définitivement de la tuberculose; il exerce le métier de pressier sur une chaîne de production d'arbres de Noël en aluminium, chez Home Pool Equipment, une usine située en Californie ${ }^{16}$. Il occupe d'autres emplois en usine, en Californie puis dans l'État de New York, avant que le succès lui permette de quitter définitivement les métiers industriels.

Ces artistes résident dans des villes régionales importantes (Drummondville, Saint-Hyacinthe, Granby) qui leur permettent, en début de carrière, d'exercer à la fois un métier industriel et celui de

7 Yves Claudé, «Le country-western au Québec, structures sociales et symboliques », dans Paul Bleton et Richard Saint-Germain (dir.), Les hauts et les bas de l'imaginaire western dans la culture médiatique, Montréal, Tryptique, 1997, p. 171.

8 Roger Chamberland, "La chevauchée "lyrique" de la musique western ", dans Bleton et Saint-Germain (dir.), Les hauts et les bas de l'imaginaire western dans la culture médiatique, p. 209.

9 Le Serge, Willie Lamothe, p. 54.

${ }_{10}$ Ibid., p. 15.

11 Godin, "Ils ont inventé le cowboy québécois », p. 39.

12 Martel et Boulanger, Au jardin de mes souvenirs, p. 73; Gendron, "Paul Brunelle».

${ }_{13}$ Martel et Boulanger, Au jardin de mes souvenirs, p. 34.

14 Ibid., p. 60.

15 Ibid., p. 71-72.

16 Ibid., p. 194-195. 
chanteur dans le cadre de fêtes privées ou communautaires ainsi que dans les hôtels de la région. Un réseau de petites salles de spectacles partout en province (cabarets, hôtels, salles paroissiales et sous-sols d'église) permet à ces artistes de se faire connaître et d'engranger des revenus considérables pendant la saison estivale. Ils sont très présents sur les ondes et dans les studios d'enregistrement, et une partie non négligeable de leur carrière médiatique se déroule dans la métropole. Roland Lebrun et Marcel Martel enregistrent chez Compo sur étiquette Starr, Paul Brunelle et Willie Lamothe chez RCA Victor, deux compagnies qui ont pignon sur rue à Montréal et qui possèdent également des installations en périphérie immédiate (Lachine pour Compo, Saint-Henri pour RCA). Pendant les années 1950, Brunelle et Lamothe jouissent d'une présence soutenue dans les studios de radio de la métropole, dont CKAC, CHLP (Montréal) et CKVL (Verdun). C'est en partie grâce à cette présence urbaine, notamment dans la métropole, que les artistes country-western ont pu se professionnaliser, un processus qui s'est accompli, entre autres, grâce à la radio.

\section{La radio}

La musique country est née dans les années 1920 aux États-Unis et elle est issue de la commercialisation de la musique de tradition orale telle que pratiquée par les musiciens amateurs des États du Sud-Est. Il s'agit sans doute de la formulation la plus simple de ce qui est en fait un phénomène complexe, formulation à laquelle il faut déjà apporter trois nuances. D'abord, le répertoire de ce qu'on a appelé old-time music ou hillbilly music n'était que partiellement constitué de musiques et de chansons d'origine folklorique; les adaptations de chansons de la Tin Pan Alley, les chansons originales et les spirituals y étaient nombreux ${ }^{17}$, et le style instrumental empruntait autant au

17 Dans une recension des enregistrements de musique hillbilly effectués par la compagnie Columbia dans sa série 15000-D entre 1925 et 1931, Charles Wolfe note que le répertoire qu'on y trouve est composé de musique traditionnelle 
folklore anglo-celtique qu'au blues, au jazz, à la musique hawaïenne et à la musique religieuse. Ensuite, si ce répertoire était principalement transmis par des moyens relevant de l'oralité et, de manière négligeable, par l'écriture et la partition - la musique en feuilles -, le disque et les rouleaux de piano mécanique ont largement favorisé la pénétration en région rurale d'un vaste répertoire urbain qui était en partie passé dans la tradition country ${ }^{18}$. Enfin, comme l'explique Richard Peterson, le statut professionnel des artistes country était complexe. Ces derniers étaient pour la plupart exclus d'emblée de l'American Federation of Musicians (AMF), qui imposait à ses futurs membres un test d'admission de lecture à vue (évaluant leur capacité à lire une partition de façon immédiate). De plus, l'American Society of Composers, Authors, and Publishers (ASCAP) exerçait un quasimonopole sur l'édition de la musique en feuilles et sur le répertoire diffusé dans les théâtres et les salles de concerts. Les artistes country ne peuvent donc, dans les années 1920, accéder facilement au statut de musicien professionnel. Ces derniers étaient cependant très présents dans la vie musicale urbaine et rurale des États du Sud-Est, lors de fêtes municipales, religieuses ou privées, de rassemblements politiques, de medicine shows, de concours musicaux ou encore d'ouverture de commerces ${ }^{19}$. Les musiciens country sont donc des amateurs dans le sens où ils n'ont, pour la plupart, jamais reçu de formation musicale en dehors de la transmission orale, de pair à pair, et qu'ils ne sont pas considérés, dans l'industrie de la musique, comme des professionnels. La musique qu'ils pratiquent est cependant largement diffusée dans la région des Appalaches. Certains participent régulièrement à des concours régionaux ou nationaux, et

dans une proportion de 33,4\% (Charles K. Wolfe, "Columbia Records and Old-Time Music ", John Edwards Memorial Foundation Quarterly, vol. 14, n 51 (automne 1978), p. 121).

18 Bill C. Malone trace un portrait détaillé des diverses sources musicales à l'origine du style et du répertoire country dans le premier chapitre de Country Music, U.S.A., Austin, University of Texas Press, 2002, p. 1-29.

19 Peterson, Creating Country Music, p. 13. 
si plusieurs occupent un emploi ouvrier ou agricole, ils sont des musiciens qu'on rémunère pour leurs services. La radio va contribuer à leur professionnalisation et à l'intégration de la musique country dans une industrie musicale structurée.

\section{Radio et musique country : destins liés}

Le développement de la musique country est étroitement lié à celui de la radiodiffusion commerciale. Pour les artistes, il s'agit d'un moyen efficace de joindre un public plus nombreux et de rayonner à l'extérieur de leur localité. Pour les promoteurs, les artistes country constituent une source inépuisable de contenu à bon marché ${ }^{20}$. De plus, les opérateurs de stations de radio se rendent rapidement compte que ces artistes rencontrent une grande faveur populaire :

In the first several months of its existence WBAP [Fort Worth, Texas]as was true of almost all early radio stations_programmed nothing but popular, jazz, sacred, and semiclassical music. But on the night of January 4, 1923, WBAP featured an hour-and-a-halfprogram of square dance music directed by an old-time fiddler and Confederate veteran Captain M. J. Bonner. The music was performed by a string band which normally played only Hawaiian music (Fred Wagner's Hilo Five Hawaiian Orchestra), and Captain Bonner's was the only fiddle music provided. The program provoked the largest audience response - in the form of telegrams and telephone calls - that the station had experienced in its short history. Touching an unexploited but eager audience, WBAP thus inangurated the hillbilly radio barn dance ${ }^{21}$.

Les stations de radio voient rapidement l'avantage de répondre aux préférences du public. En retour, la diffusion à l'échelle nationale d'artistes d'origine rurale ou qui viennent de petites villes du Sud contribue à transformer le goût des Américains ${ }^{22}$, à une époque où

${ }^{20}$ Malone, Country Music, U.S.A., p. 32.

${ }^{21}$ Ibid., p. 33-34.

22 Ibid., p. 32. 
la production de la musique populaire, sous l'égide de l'ASCAP, était concentrée dans quelques grandes villes du Nord.

Vingt ans plus tard, des phénomènes semblables contribueront à l'émergence de la chanson country-western au Québec où, comme aux États-Unis, la radio se propage rapidement. Au début des années 1940, elle est déjà présente dans plus de $70 \%$ des foyers québécois ${ }^{23}$, surpassant la pénétration du disque, une technologie qui l'avait pourtant précédée de quelques décennies. Si $88 \%$ des familles possèdent un appareil radio en $1947^{24}$, Albert Lévesque évalue à $90 \%$ le nombre de foyers appartenant à l'élite canadienne-française qui possèdent un récepteur en $1944^{25}$. Avant la création du Conseil de la radiodiffusion et des télécommunications canadiennes (CRTC) en 1968, les Québécois avaient accès à la radio américaine et à la musique country diffusée sur ses ondes ${ }^{26}$. La programmation de WBAP, par exemple, pouvait être captée à New York, au Canada, à Hawaï et même en Haïti ${ }^{27}$. Les émissions de WLS à Chicago (WLS Barn Dance, 1924) et de WSM à Nashville (Grand Ole Opry, 1925) peuvent être entendues dans plusieurs régions du pays ${ }^{28}$. Au Québec, la station CKAC, qui est affiliée à la géante CBS, diffuse des émissions produites par le diffuseur national. Marcel Martel se souvient y avoir

${ }^{23}$ Dans un article portant sur l'histoire de la radio au Canada français, Elzéar Lavoie s'est penché sur le recensement de 1941, lequel indique que le Québec compte alors $25 \%$ des ménages canadiens et $22,5 \%$ des récepteurs radio du Canada. C'est sur ces données que Lavoie fonde son calcul : 70,6\% des ménages québécois possèdent alors un récepteur radio (Elzéar Lavoie, "L'évolution de la radio au Canada français avant 1940 ", Recherches sociographiques, vol. 12, n 1 (1971), p. 25-26).

${ }^{24}$ Paul-André Linteau et al., Histoire du Québec contemporain, t. 2 : Le Québec depuis 1930, Montréal, Éditions du Boréal, 1989, p. 176.

25 Albert Lévesque, "Entrez donc " : analyse du comportement familial de la population de langue française, au Canada: première étape : sentiments et intérêts de l'élite, Montréal, Albert Lévesque, 1944, p. 121.

${ }^{26}$ Chamberland, "La chevauchée "lyrique" de la musique western ", p. 209.

27 Malone, Country Music, U.S.A., p. 34.

28 Richard Green, "Musique country ", dans Encyclopédie canadienne, dernière modification : 6 avril 2014, [En ligne], [http://www.encyclopediecanadienne. $\mathrm{ca} /$ fr/article/musique-country/] (2 mai 2016). 
entendu pour la première fois de la guitare à la radio et qu' il est « fort possible que ce soit Jimmy Rodgers $[\text { sic }]^{29}$ ".

La radio joue un rôle fondamental dans l'émergence du countrywestern au Québec, dont les premiers artistes y font pour la plupart leurs débuts médiatiques, bien avant de passer dans les studios d'enregistrement. À la suite de sa victoire au concours d'amateurs du Palais Montcalm, Roland Lebrun est invité à se produire à la station CHRC de Québec. La popularité de son premier succès, L'adieu du soldat, précède la production du disque qu'il enregistre en février 1942. Un mois avant, une lectrice de La Patrie demande les paroles de la chanson $^{30}$, et la station CHRC aurait reçu plusieurs centaines de demandes à la même période ${ }^{31}$. En 1942 et en 1944, Paul Brunelle remporte un concours d'amateurs présenté en direct sur les ondes de CKAC, concours dont il sera question plus loin; c'est à la suite de ces passages à la radio que RCA Victor lui offre un contrat. Willie Lamothe prend lui aussi d'abord contact avec le public par l'entremise des ondes radiophoniques. Enrôlé volontairement pendant la guerre, il divertit les troupes lors des army shows et écrit pour les soldats de nouvelles paroles sur des airs folkloriques dont il vend des exemplaires aux recrues ${ }^{32}$. Ferdinand Biondi, présentateur et directeur artistique à CKAC, entend Willie Lamothe lors d'un de ces spectacles et l'invite à la radio ${ }^{33}$, ce qui, on le verra, scellera en quelque sorte l'orientation de sa carrière. Quant à Marcel Martel, il joue d'abord sur scène à Drummondville et se fait remarquer par Ovila Légaré, qui l'engage pour une courte tournée en 1942. En février 1944, il reçoit son ordre de mobilisation; au cours de l'examen médical obligatoire, on lui diagnostique une tuberculose. Il est admis au sanatorium Cook, à Trois-Rivières, où, à l'été 1944, il joue pour les autres malades lors d'une fête à laquelle assiste le directeur des

\footnotetext{
29 Martel et Boulanger, Au jardin de mes souvenirs, p. 30.

30 "Le courrier du dimanche ", La Patrie, édition finale, 18 janvier 1942, p. 19.

31 Radiomonde, 7 février 1942, p. 10.

${ }^{32}$ Le Serge, Willie Lamothe, p. 40-41.

33 Ibid., p. 43.
} 
programmes de la station locale, CHLN; ce dernier lui demande d'interpréter quelques chansons en ondes, ce qui mène le chanteur à y présenter une émission quotidienne ${ }^{34}$.

Les chanteurs country-western sont présents à la radio tout au long des années 1940 et 1950. En 1945, Paul Brunelle anime une émission sur les ondes de CKAC ${ }^{35}$. De 1955 à 1957, il anime une émission quotidienne d'une heure sur les ondes de CKVL, à Verdun, Paul Brunelle et ses troubadours du Far-West ${ }^{36}$. En 1947 et 1948, Willie Lamothe anime Willie Lamothe et ses chansons, une émission hebdomadaire présentée sur les ondes de CJSO à Sorel ${ }^{37}$. En 1950, il fait son entrée à CKVL et y anime Willie Lamothe et ses cavaliers des plaines, une émission hebdomadaire enregistrée au Café Saint-Jacques, à l'angle des rues Sainte-Catherine et Saint-Denis, qui sera diffusée pendant plusieurs années. En 1952, il obtient son deuxième engagement radiophonique à CKAC et y présente une série d'émissions intitulée $A u$ Far-West. En parallèle, il poursuit sa participation à la programmation de CKVL, où il travaillera durant dix ans, ce qui lui assure une présence prolongée et hebdomadaire sur les ondes montréalaises $^{38}$. Quant à Marcel Martel, il est engagé en 1946 par la station CHEF de Granby où, en alternance avec Roland Tétrault (né en 1917), il chante en direct les demandes du public à 6 heures du matin $^{39}$. La carrière médiatique des premiers artistes country-western se déroule donc à la fois dans leur ville et leur région d'origine et dans la métropole. CHLP, une autre station montréalaise, alors propriété du journal La Patrie, fait une place au country-western dans ses émissions généralistes dès la fin des années 1940. En 1947, Maurice

\footnotetext{
Martel et Boulanger, Au jardin de mes souvenirs, p. 51-52.

35 Gendron, "Paul Brunelle ».

36 Roger Charlebois, Super festival western 1976, [Montréal], [Bonanza], [1976], n. p.

37 Mario Gendron, "Willie Lamothe ", Portraits de la culture montérégienne : la chanson western, sur le site Chanson d'expression française, [http://www.archivessh. qc.ca/culture/frameset_chanson.html] (23 mai 2016).

${ }^{38}$ Le Serge, Willie Lamothe, p. 75-77.

39 Martel et Boulanger, Au jardin de mes souvenirs, p. 71-72.
} 
Bienvenue, sous le pseudonyme de Jimmy Debate, y anime son émission hebdomadaire ${ }^{40}$. Le samedi, une case horaire est réservée à un " orchestre vedette ", et le samedi 3 janvier 1948, on y annonce la venue de Curley Hachey (le frère de Bobby Hachey) and His Sunset Playboys ${ }^{41}$.

On le verra, des chanteurs comme Roland Lebrun, Marcel Martel, Willie Lamothe et Paul Brunelle ont des parcours individuels variés et n’ont pas nécessairement choisi de faire carrière en tant qu'artistes country-western. Ce sont des conditions diverses et complexes qui ont mené à la structuration d'un genre musical qui deviendra somme toute relativement homogène à mesure qu'il se développe. Une chose est sûre, la radio joue un rôle important dans la conjoncture qui permet au country-western de rayonner. En premier lieu parce qu'elle fait une place importante aux amateurs et parce qu'elle ouvre une voie de communication ininterrompue entre les artistes et le public.

\section{L'ère des amateurs}

Dès les débuts de la radiodiffusion commerciale, les stations intègrent les artistes amateurs dans leur programmation. Le cas de CKAC est sans doute l'un des plus intéressants. Propriété du journal La Presse, l'entreprise commence ses activités en 1922 sous la houlette de Jacques-Narcisse Cartier, qui a l'ambition d'offrir aux auditeurs une programmation de haute qualité et de faire de la station un instrument d'éducation populaire. L'éducation musicale et la diffusion des artistes canadiens-français sont au cour de la mission de CKAC. En 1925, le pianiste Emiliano Renaud lance une série de trente leçons de piano en direct, accompagnées d'un manuel vendu par La Presse ${ }^{42}$. Cartier organise, en 1923, la présentation en direct de l'opérette

\footnotetext{
40 "1947, mille raisons d'écouter CHLP », La Patrie, 5 janvier 1947, p. 59.

41 "CHLP présente", La Patrie, 3 janvier 1948, p. 34.

42 Pierre Pagé, Histoire de la radio au Québec : information, éducation, culture, Montréal, Éditions Fides, 2007, p. 212.
} 
Les cloches de Corneville avec orchestre, solistes et chœur, et la station diffuse abondamment des œuvres du grand répertoire tout en faisant la promotion des artistes canadiens. Mais CKAC propose aussi « un éventail d'émissions permettant au grand public de se retrouver dans ses pratiques culturelles familières ${ }^{43}$ ". La station présente, notamment, au cours des années 1920, des concerts d'harmonies municipales et régionales ainsi qu'un concours national visant à récompenser les meilleures fanfares, municipales ou scolaires, qui se déplaçaient pour offrir des concerts en direct, souvent accompagnées par le maire de leur village, une initiative qui rassemble des musiciens amateurs de l'ouest et du centre du Québec ${ }^{44}$.

Le travail d'Yvonne Duckett, mieux connue sous le nom de Madame Audet, se situe lui aussi à la frontière de l'éducation populaire et de l'appui à la pratique musicale amateur. De 1936 à 1950, cette professeure de phonétique, d'élocution et de technique vocale anime Radio Petit-Monde ou une de ses variantes, sur les ondes de CRCM, CHLP, CKAC et SRC consécutivement ${ }^{45}$. L'émission met en vedette ses jeunes élèves dans des saynètes et des dialogues. Yvonne Duckett enseignait dans les écoles et les couvents et donnait aussi des cours privés. Certains de ses élèves mèneront une carrière artistique professionnelle. Mais Yvonne Duckett désirait avant tout rejoindre le plus grand nombre d'enfants possible; elle ne refusait aucun élève, quelle que soit la situation financière de ses parents et, selon son fils JeanMarc, elle accueillait gratuitement $75 \%$ de ses élèves ${ }^{46}$. Ces initiatives mettant en valeur des amateurs s'inscrivaient dans une culture artistique et musicale encore très axée sur la pratique. Les revues culturelles comme La Lyre et Le Passe-Temps proposent à chaque

\footnotetext{
43 Ibid., p. 350.

44 Ibid., p. 350-351.

45 Muriel Gold, "A Missionary of the French Language Through Drama: Madame Jean-Louis Audet (1890-1970) ", Recherches théatrales au Canada, vol. 12, n 1 (printemps 1991), [En ligne], [http://journals.hil.unb.ca/index.php/tric/article/ view/7277/8336] (15 mai 2016).

46 Ibid.
} 
numéro, jusque dans les années 1920 et 1930, des partitions à leurs lecteurs. Ces revues sont elles aussi très préoccupées par l'éducation musicale des amateurs.

Dans les années 1940, les émissions consacrées aux amateurs sont de plus en plus populaires et plusieurs prennent désormais la forme de concours. Dans L'Action nationale de février 1947, Jacques Beauchamp s'attaque à cet engouement dans un article intitulé " Un peuple a la radio qu'il veut ». Il collige plusieurs renseignements publiés dans Radiomonde, qui révèlent le goût du public radiophonique des années 1940 pour les émissions mettant en vedette des amateurs. Ainsi, dans les années 1944 et 1946, l'émission la plus populaire est En chantant dans le vivoir, qui diffuse sur les ondes de CKAC le concours commandité par la Living Room Furniture, celuilà même remporté à deux reprises par Paul Brunelle, et qui est écoutée par 35,9\% des auditeurs ${ }^{47}$. L'émission, animée par Bernard Goulet, est diffusée depuis le Théâtre Château, où le concours se déroule devant public ${ }^{48}$. Les talents de chez nous, qui diffuse des performances d'artistes amateurs, figure au palmarès des dix émissions les plus écoutées en 1946. Selon Beauchamp, qui déplore la situation : «Les programmes dits "d'amateurs" restent l'une des formules que le public apprécie le plus. Voilà pourquoi la radio montréalaise diffuse cinq soirées d'amateurs ${ }^{49}$. "

Les initiatives de CKAC et d'Yvonne Duckett mettaient en valeur des pratiques artistiques collectives dans des formules fixées d'avance et très normées. Au contraire, ces nouvelles émissions d'amateurs, qui prennent souvent la forme de concours, semblent davantage tournées vers la découverte de talents singuliers. L'idée qu'il soit possible pour quiconque de devenir un artiste de la radio, voire une vedette, semble émerger dans les années 1930. En 1931, le Studio

47 Jacques Beauchamp, "Chronique de la radio : un peuple a la radio qu'il veut ", L'Action nationale, vol. 29, $\mathrm{n}^{\circ} 2$ (février 1947), p. 155-161.

48 "Bernard Goulet et les amateurs", Radiomonde, 24 janvier 1942, p. 13.

49 Beauchamp, "Chronique de la radio », p. 156. 
de musique hawaïenne, sis rue Bélanger, à Montréal, annonce ses leçons de ukulélé, de guitare hawaïenne et de guitare espagnole dans les pages du Passe-Temps, en affirmant que les leçons offertes par l'établissement " préparent l'élève à tous les avantages qu'offrent l'art et la popularité : tels que soirées, Radio, etc. ${ }^{50}$ ". Les années 1940 voient progressivement s'installer un vedettariat canadien-français, et le public aspire à y prendre part. Ainsi, Photo-Journal publie, en 1948, un article intitulé "Voulez-vous devenir vedette de la chansonnette? Jeunes filles, jeunes femmes douées, lisez ceci ${ }^{51}$ ", qui propose à ses lectrices "quelques conseils et quelques "ficelles" ". Le succès y est présenté comme étant relativement accessible et passant par la radio :

Car une chose est d'épater ses petites cousines, et une autre chose est de subjuguer le grand public de CKVL. [...] Quand vous serez prête, vous pouvez demander à passer une audition à la radio. On $\mathrm{y}$ entend tout le monde, et si votre voix et votre genre plaisent, vous êtes convoquée pour une émission d'essai. Depuis un an, beaucoup de jeunes artistes sont sortis ainsi grâce à la radio ${ }^{52}$.

Ce sont précisément des concours d'amateurs qui permettent à Roland Lebrun et à Paul Brunelle d'être découverts. Marcel Martel et Willie Lamothe sont eux aussi des amateurs au sens où ils n'ont pas reçu de formation musicale. Il apparaît que la large place laissée aux musiciens et aux chanteurs amateurs, la grande popularité des concours qui s'adressent à eux ainsi que, sans doute, la nécessité de fournir des artistes toujours plus nombreux pour combler des dizaines d'heures de programmation hebdomadaires ont favorisé l'éclosion de carrières d'un genre nouveau.

50 Publicité pour le Studio de musique hawaïenne, Le Passe-Temps, no 842 (1931), p. 116.

51 Roger Malher, "Voulez-vous devenir vedette de la chansonnette? Jeunes filles, jeunes femmes douées, lisez ceci ", Photo-Journal, 26 août 1948, p. 37.

52 Ibid. 


\section{Un lieu de rencontre avec le public}

La radio a permis d'établir une relation de grande proximité entre les artistes de la radio et leurs auditeurs, les premiers pouvant pénétrer en direct dans l'univers domestique et intime des seconds. Le crooner, souvent à la fois animateur et chanteur, est sans doute le spécimen par excellence de l'artiste de la radio mettant en œuvre diverses stratégies qui donnent l'impression de s'adresser personnellement à chaque auditeur, notamment grâce à un usage particulier du microphone et de la voix. Les chanteurs country des États-Unis, dont certains d'entre eux étaient aussi animateurs, usaient de tels procédés, rendus explicites dans cette préface à un recueil de chansons publié par Bradley Kincaid, qui a amorcé sa carrière à la radio en 1926 :

When I sing for you on the air, I always visualize you, a family group, sitting around the table of the radio, listening and commenting on my program. Some of you have written and said that I seem to be talking right to you, and I am. If I did not feel your presence, though you be a thousand miles away, the radio would be cold and unresponsive to me, and I in turn would sound the same way to you $u^{53}$.

On possède peu d'information sur la manière dont les chanteurs country-western envisageaient et exerçaient leurs fonctions d'animateurs. On sait cependant que les premiers engagements radiophoniques de Marcel Martel et de Willie Lamothe consistaient à répondre aux demandes du public. À CJSO (Sorel), Lamothe est seul à la guitare; il interprète ses chansons et " dédicace » des disques, ce qui revenait sans doute à faire jouer les demandes spéciales ${ }^{54}$. Marcel Martel, on l'a vu, interprète en direct les requêtes du public

53 Kristine M. McCusker, " "Dear Radio Friend”: Listener Mail and the National Barn Dance, 1931-1941 ", American Studies, vol. 39, nº 2 (été 1998), p. 179, cité dans Timothy D. Taylor, « Music and the Rise of Radio in Twenties America: Technological Imperialism, Socialization, and the Transformation of Intimacy ", dans Paul D. Greene and Thomas Porcello (dir.), Wired For Sound: Engeneering and Technology in Sonic Cultures, Middletown, Wesleyan University Press, 2005, p. 262.

${ }^{4}$ Le Serge, Willie Lamothe, p. 71-72. 
sur les ondes de CHEF en 1946. À l'été 1944, lorsqu'il fait ses débuts à CHLN, à l'invitation du directeur de la station, le chanteur se rend tous les après-midis au studio et y interprète en direct les demandes spéciales des auditeurs, très souvent des chansons du soldat Lebrun, qui sont dédiées aux malades des autres hôpitaux de Trois-Rivières. Comme le résument Martel et Boulanger, un «malade qui chante pour des plus malades ${ }^{55}$ ".

Les requêtes du public et l'engouement des auditeurs pour certains types d'émissions contribuent à modeler le contenu radiophonique, et ce, pratiquement dès les débuts de la radiodiffusion commerciale. Grâce à la radio, "les goûts et les sensibilités de la population s'imposaient face au discours d'autorite ${ }^{56}{ }^{\prime}$. Cette attention portée au public a révélé non seulement les goûts de celui-ci, mais aussi son fractionnement en plusieurs groupes : «Il fallait plaire à la clientèle; très tôt, aux clientèles ${ }^{57}$. " Marcel Martel raconte d'ailleurs comment, après trois semaines à CHLN, la durée de son émission quotidienne passe de quinze à trente minutes, le deuxième quart d'heure étant consacré aux malades d'un hôpital qui avait " fait les pressions nécessaires pour avoir du temps d'antenne ${ }^{58}$ ".

Ce mécanisme ne semble cependant pas toujours être allé de soi, comme en témoignent quelques numéros du Passe-Temps. De 1933 à 1935, cette revue artistique et littéraire, sous l'impulsion de son nouveau propriétaire, Eddy Prévost, s'intéresse particulièrement à la radio et à son contenu. Les qualités et les mérites des émissions, des artistes, des animateurs font l'objet d'une véritable critique, et on interpelle le public qui, selon les éditeurs, a un rôle important à jouer dans l'élaboration des programmes radiophoniques :

Le meilleur moyen pour les radiophiles d'obtenir ce qu'ils désirent, c'est d'écrire aux postes de radio, et de ne pas craindre d'exprimer

\footnotetext{
55 Martel et Boulanger, Au jardin de mes souvenirs, p. 52.

56 Jean Du Berger, Jacques Mathieu et Martine Roberge, La radio à Québec, 19201960, Québec, Les Presses de l’Université Laval, 1997, p. 12.

57 Ibid., p. 13.

58 Martel et Boulanger, Au jardin de mes souvenirs, p. 52.
} 
leur approbation... ou leur mécontentement au besoin. Contrairement au théâtre, les directeurs des postes de radio n'ont pas une assistance visible pour constater le réflexe des auditeurs. Les lettres remplaçant les applaudissements... ou les sifflets ${ }^{59}$.

Le Passe-Temps publie même un encadré intitulé "Comment écrire à un poste de radio ", dans lequel on précise qu'un "grand nombre de radiophiles négligent d'écrire aux postes de Radio car ils n'en connaissent pas l'adresse exacte ${ }^{60} »$. Dix ans plus tard, l'habitude semble bien ancrée chez les auditeurs. En 1942, Radiomonde publie :

Un des artistes récemment engagés par CHRC qui aura fait le plus de sensation, c'est le soldat Roland Lebrun qui chante à la manière "Cow Boy", s'accompagnant sur une guitare. Ce jeune militaire a composé et créé une chanson intitulée «L'Adieu du Soldat ». Dans les quelques semaines qui suivirent cette création, Gaston Voyer enregistra la réception d'au-delà de 1500 demandes spéciales d'auditeurs et auditrices qui voulaient re-entendre $[s i c]$... encore, et encore... "L'Adieu du Soldat ${ }^{61}$.

Par la suite, la station CHRC consacre une émission à Roland Lebrun le lundi soir ${ }^{62}$ et, en 1943, elle doit engager une employée dont la tâche consiste exclusivement à traiter la correspondance du chanteur $^{63}$. Un journaliste remarque un engouement similaire du public pour Willie Lamothe, lui prédisant cependant une bien courte carrière :

Dès qu'on tourne le bouton d'un poste de radio on nous rabat [sic] les oreilles des chansons de Willie Lamothe, cette voix de crécelle; quinze ou vingt fois par jour les gens exigent que l'on fasse jouer ses chansons. Mais nous sommes persuadés que son

\footnotetext{
59 "Allan McIver : le boute-en-train de la radio à Montréal ", Le Passe-Temps, vol. 40, no 876 (août 1934), p. 44, [En ligne], [http://collections.banq.qc.ca/ ark:/52327/2273412].

${ }^{60}$ Ibid.

${ }^{61}$ Radiomonde, 7 février 1942, p. 10.

${ }_{62}$ Du Berger, Mathieu et Roberge, La radio à Québec, p. 154.

${ }^{63}$ Ibid., p. 70.
} 
succès ne sera que de courte durée et que très bientôt nous n'en entendrons plus parler... ${ }^{64}$

À CJSO, le chanteur reçoit entre 500 et 600 lettres par semaine ${ }^{65}$. Il en reçoit aussi à la maison, et son épouse, devant l'abondance du courrier, décide de vendre, par correspondance, des photos de son mari à 10 cents l'unité. Elle affirme avoir reçu plus de 20000 demandes en quelques mois ${ }^{66}$.

Au moment où les chanteurs country-western font leurs débuts à la radio, l'habitude d'écrire à une station de radio est donc bien ancrée chez le public. Ce dernier semble d'ailleurs avoir joué un rôle majeur dans l'émergence même du genre. Au départ, Paul Brunelle interprétait plusieurs chansons de Tino Rossi et de Bing Crosby; c'est RCA qui lui a demandé de chanter dans le style country-western, dans le but explicite de concurrencer Roland Lebrun ${ }^{67}$. Willie Lamothe, quant à lui, a décidé très jeune de tenter sa chance dans le monde du spectacle, d'abord comme comédien amateur, puis comme danseur et professeur de danse. Ce sont ses performances exécutées dans les army shows qui lui ont permis de se faire remarquer par Ferdinand Biondi puis de chanter à CKAC. Son répertoire de l'époque semble avoir été constitué de chansons traditionnelles dont il transformait, à l'occasion, les paroles. Grand admirateur de Maurice Chevalier, il interprétait plusieurs de ses chansons. C'est en quelque sorte l'engouement des auditrices de CKAC qui l'amène à adopter l'attirail de cow-boy, alors qu'il se produit encore sous le surnom de "sergent chantant", en hommage au soldat Lebrun. Son épouse raconte les réactions suscitées par ses présences sur les ondes de cette station en 1943 : «Willie se découvrit alors des marraines de guerre qui lui faisaient parvenir des photos, des colis et même de l'argent quand ce n'était pas des lettres d'amour enflammées. [...] Il avait

\footnotetext{
${ }^{64}$ Le Serge, Willie Lamothe, p. 65.

65 Ibid., p. 72.

${ }^{66}$ Jeannette Lamothe, Mes 45 ans avec Willie Lamothe, Montréal, 7 jours, 1991, p. 68.

67 Godin, «Ils ont inventé le cowboy québécois », p. 40.
} 
enfin trouvé sa voie. Jamais plus il n'allait déroger de ce domaine qui lui avait apporté ses premiers véritables succès ${ }^{68}$. "Le chanteur conquiert alors de nombreuses admiratrices et l'une d'elles lui confectionne son premier costume de cow-boy ${ }^{69}$. Il prend conscience également de l'engouement pour les chansons western après son retour à la vie civile, lors d'un spectacle donné avec son ami Victor Martin, un violoniste de folklore, pour les commis-voyageurs de RCA Victor. Après avoir joué quelques reels et chanté des chansons connues devant un public tiède, on lui demande s'il peut faire des chansons western. Willie Lamothe, qui en avait composé une, Au loin dans ma vallée, l'interprète alors avec succès. Il raconte que c'est à ce moment qu'il a eu la certitude d'avoir enfin trouvé un créneau qui lui convenait ${ }^{70}$. Quant à Marcel Martel, il chantait, au commencement de sa carrière, des chansons de Tino Rossi et du soldat Lebrun, de même que des chansons country américaines ${ }^{71}$. C'est grâce à l'enregistrement sonore que le style des artistes country-western allait s'uniformiser.

\section{Les studios d'enregistrement}

Les circonstances qui ont permis aux premiers chanteurs countrywestern d'accéder aux studios d'enregistrement sont variées. Selon l'historien Robert Thérien, c'est l'armée canadienne qui aurait " arrangé » le contrat de disque de Roland Lebrun ${ }^{72}$. L'hypothèse est très plausible. Le chanteur est en effet assigné aux services auxiliaires de l'armée canadienne et il amorce, à la suite de son passage remarqué à CHRC, une série de tournées au Québec et partout au Canada pour présenter ses chansons dans les bases militaires, à la radio et sur scène pendant trois ans ${ }^{73}$, effectuant un véritable travail de propa-

${ }_{68}$ Lamothe, Mes 45 ans avec Willie Lamothe, p. 46.

69 Ibid.; Le Serge, Willie Lamothe, p. 43.

70 Le Serge, Willie Lamothe, p. 49.

${ }_{71}$ Martel et Boulanger, Au jardin de mes souvenirs, p. 38-40.

${ }^{72}$ Robert Thérien, L'histoire de l'enregistrement sonore au Québec et dans le monde, 1878-1950, Québec, Les Presses de l’Université Laval, 2003, p. 205.

73 Robert Thérien, "Le soldat Roland Lebrun ", notes pour Le Soldat Lebrun : les années Starr, 1942-1953, disques XXI XXI-CD 2 1501, 2004, p. 6. 
gande pour l'armée et pour la conscription. La chanson gravée sur la face $\mathrm{A}$ de son premier disque le 7 février $1942^{74}$ est déjà un succès, et les ventes auraient été "phénoménales ${ }^{75}$ ». Paul Brunelle est remarqué par A. Hugh Joseph, alors Artist and Repertoire Manager ( $A \& R$ man) chez RCA Victor, lors de sa seconde victoire au concours de la Living Room Furniture en $1944^{76}$; la compagnie, on l'a vu, voyait en lui l'artiste qui pouvait concurrencer le succès de Roland Lebrun chez Starr, et fait paraître son premier disque en 1945. Willie Lamothe se produit déjà en public depuis quelques années lorsqu'il rencontre Victor Martin, qui enregistre chez RCA Victor; c'est Martin qui lui obtient un premier essai en studio ${ }^{77}$ le 13 juin $1946^{78}$. La même année, Starr commence à enregistrer les chansons de PaulÉmile Piché et de Georges Caouette, deux chanteurs countrywestern. Caouette recommande à Marcel Martel d'approcher la compagnie à son tour; il sera invité à la première session d'enregistrement en octobre $1947^{79}$.

Lebrun, Martel, Brunelle et Lamothe enregistrent plusieurs disques au cours des années 1940 et 1950, ce qui exige d'eux une présence régulière à Montréal. Si c'est la radio qui a contribué à lancer les carrières des artistes country-western, tout en jouant un rôle de premier plan dans la diffusion de leur musique, le disque assure à ces derniers une certaine stabilité en leur fournissant des revenus importants et réguliers. Certaines données indiquent que les enregistrements des chanteurs country-western étaient lucratifs tant pour les chanteurs eux-mêmes que pour les compagnies de disques. Pour le disque Je chante à cheval/ Ma destinée, Willie Lamothe aurait touché 400 \$

${ }^{74}$ Le jour même de l'enregistrement du disque, la chanson fait l'objet d'un entrefilet dans Radiomonde. L'article indique que la chanson était déjà connue du public, qui l'avait entendue à la radio (voir la note 61).

75 Thérien, «Le soldat Roland Lebrun ", p. 6.

76 Thérien, L'histoire de l'enregistrement sonore au Québec et dans le monde, p. 207.

77 Lamothe, Mes 45 ans avec Willie Lamothe, p. 20.

78 Gendron, "Willie Lamothe".

79 Martel et Boulanger, Au jardin de mes souvenirs, p. 73. 
de droits d'auteur dans les trois premiers mois de sa mise en marché ${ }^{80}$. Ses deux premiers disques lui rapportent rapidement 2500 \$ en droits d'auteur, un montant plus élevé que son salaire annuel à l'usine Goodyear $^{81}$, où il occupe pourtant un poste de contremaître ${ }^{82}$. Les redevances sont suffisantes pour lui permettre de quitter son emploi permanent. Le succès commercial des enregistrements de Lamothe incite RCA Victor à lui offrir un contrat de trois ans; en 1947, la compagnie lance un nouveau disque de Willie Lamothe tous les trois mois ${ }^{83}$. Les redevances, qui pendant quelque temps continuent de " monter en flèche ", vont constituer, en plus des recettes provenant de quelques spectacles, la totalité des revenus du chanteur avant l'époque des tournées ${ }^{84}$. Quant à Marcel Martel, les ventes de disques constituent pour lui aussi un revenu important. Elles ne lui assurent pas la richesse, mais sont assez substantielles et lui permettent de "vivre au cours de ces années où les salaires hebdomadaires des employeurs n'entraient pas régulièrement ${ }^{85}$ ". Dès la sortie de son premier disque, qui comprend les chansons « La chaîne de nos cœurs " et "Souvenirs de mon enfance », le public est au rendez-vous :

Les mois qui ont suivi la sortie du disque ont été pleins de promesses. Les premiers chèques de redevance sont entrés. Je me souviens qu'à l'arrivée du premier chèque au montant de $165 \$$, nous avons célébré ça en changeant tout le mobilier de la maison. [...] Compo me payait aux trois mois. Souvent, on avait hâte de voir arriver le chèque des droits d'auteur pour payer le loyer ${ }^{86}$.

Les relations de Marcel Martel avec sa maison de disques indiquent elles aussi qu'il était un atout important pour Starr. Le chanteur estime qu'il est l'artiste qui a eu le plus souvent accès au studio de

${ }^{80}$ Lamothe, Mes 45 ans avec Willie Lamothe, p. 68.

81 Le Serge, Willie Lamothe, p. 54.

82 Ibid., p. 44.

${ }^{83}$ Ibid., p. 53-54.

${ }^{84}$ Ibid., p. 56.

${ }_{85}$ Martel et Boulanger, Au jardin de mes souvenirs, p. 89.

${ }^{86}$ Ibid., p. 82. 
Compo entre 1948 et $1956^{87}$. Qu'on prenne au sérieux ou non cette affirmation, les passages en studio correspondaient à une demande provenant directement du marché puisque Marcel Martel n'avait pas signé de contrat avec la maison de disques, qui l'invitait en studio quand le besoin se faisait sentir ${ }^{88}$. Après la parution de son premier disque, les commandes, qui, selon Marcel Martel, " affluent chez Compo ", donnent lieu à une deuxième séance d'enregistrement quelques mois plus tard ${ }^{89}$. Avec Marcel Martel, Starr peut graver plusieurs disques à la fois et les mettre en marché ultérieurement, ou encore faire paraître simultanément plusieurs disques d'un même artiste, comme la compagnie le faisait avec le soldat Lebrun en $1943^{90}$. Souffrant de tuberculose, Marcel Martel avait une santé précaire et, entre les périodes où il pouvait exercer son métier de chanteur sans trop de difficulté, il connaissait des rechutes qui pouvaient l'amener à des hospitalisations prolongées. À partir du 17 avril 1950, il séjourne au sanatorium Cook pendant neuf mois. Durant ce séjour, il se rend à Montréal à deux reprises pour enregistrer de nouveaux titres ${ }^{91}$. Puis, son état de santé s'étant détérioré, le chanteur est transféré à l'hôpital de Cartierville, où il reçoit des soins plus spécialisés pendant dix-huit mois. Sa production, selon ses dires, " accuse des retards ", et la compagnie demande de nouvelles chansons. Lors d'un de ces séjours à l'hôpital, la compagnie Compo, qui voulait absolument lui faire enregistrer de nouvelles chansons, organise son transport de l'hôpital au studio :

Quelle équipée s'était [sic] quand les gens d'Apex ${ }^{92}$ ont décidé que j'allais enregistrer. Il me semblait que ça aurait été plus facile

\footnotetext{
87 Ibid., p. 90.

88 Ibid.

${ }^{89}$ Ibid., p. 82.

90 Thérien, "Le soldat Roland Lebrun », p. 6-7.

91 Martel et Boulanger, Au jardin de mes souvenirs, p. 95.

92 En 1950, le propriétaire de Compo, Herbert Berliner (1882-1966), se croyant atteint d'un cancer, vend la compagnie à Decca qui remplace l'étiquette Starr par l'étiquette Apex (Thérien, L'histoire de l'enregistrement sonore au Québec et dans le monde, p. 220), nom d'une filiale de Compo basée à Toronto.
} 
d'apporter le studio dans ma chambre... que ma chambre dans le studio. Une camionnette de déménagement de pianos est venu $[s i c]$ me chercher et les "déménageurs " m’ont traité comme un objet fragile qu'il ne faut pas casser en chemin! [...] J'ai alors réalisé ce que je représentais pour eux. Une petite fortune... [...] Le souffle était bien court et les lignes de mes chansons... trop longues. On a recommencé aussi souvent que j'étouffais. Tout le monde avait une patience d'ange avec moi. [...] Durant cette mémorable session, je n'ai enregistré que quatre chansons ${ }^{93}$.

Cinq ans plus tard, le scénario se répète, et Compo transporte le chanteur de l'hôpital au studio pour une double session d'enregistrement ${ }^{94}$.

Même si la scène et la radio, selon Robert Thérien, étaient les vrais indicateurs de succès et qu'un chanteur pouvait faire carrière en enregistrant très peu ${ }^{95}$, le contraire semble aussi vrai, car Marcel Martel a connu pendant un certain temps un grand succès fondé exclusivement sur le disque. Pendant une période particulièrement difficile sur le plan de la santé, Marcel Martel est absent de la scène durant trois ans, mais continue pourtant de connaître la faveur du public : «Il y a dans tout cela un phénomène que je ne comprends pas encore aujourd'hui et je suis quand même très reconnaissant à mon public qui continuait de m'encourager, d'acheter mes disques, même si on ne m’avait pas vu sur une scène depuis une éternité ou deux $^{96}$ ! " Pour le chanteur, c'est le disque qui est d'ailleurs perçu comme la véritable mesure du succès :

Le seul sondage valable auquel j'ai toujours cru, c'est le rapport de mes redevances : au moins là on est certain de plusieurs choses : le disque a été imprimé en tant de copies, il y en a une quantité qui a été livrée chez les marchands de musique, de ce nombre il

\footnotetext{
93 Martel et Boulanger, Au jardin de mes souvenirs, p. 104-105.

94 Ibid., p. 160.

95 Thérien, L'histoire de l'enregistrement sonore au Québec et dans le monde, p. 216.

96 Martel et Boulanger, Au jardin de mes souvenirs, p. 113.
} 
y en a qui ont été vendus... De cette quantité les marchands de musique en ont rapporté les chiffres de vente $[\ldots]^{97}$.

\section{D'un studio à l'autre : vers la professionnalisation des amateurs}

C'est au cours des années 1940 que le disque réussit véritablement à atteindre un large auditoire au Québec, alors que plus de la moitié des maisons canadiennes possèdent désormais un tourne-disque ${ }^{98}$. En même temps s'installe pour la première fois un contexte extrêmement favorable au développement d'une discographie québécoise abondante. En effet, les artistes québécois de la chanson peuvent compter sur les effets de deux conjonctures particulières qui se combinent pour stimuler l'enregistrement de chanteurs locaux. De 1941 à 1945, pendant l'occupation allemande d'une partie du territoire français, les disques produits en France ne sont plus distribués sur le marché québécois. À la pénurie de disques français s'ajoutent, dans un deuxième temps, de grands bouleversements qui affectent l'industrie américaine du disque. Afin d'obtenir des redevances pour ses membres, l'American Federation of Musicians déclenche, en 1942, une grève qui ne prendra fin qu'en 1944 et pendant laquelle les musiciens professionnels refusent de travailler dans les grands studios d'enregistrement. Entre 1940 et 1945, le disque québécois rencontre donc pour la première fois une concurrence très affaiblie ${ }^{99}$. Cette situation a suscité une forte diversification de l'industrie québécoise du disque au cours de cette décennie et a sans aucun doute favorisé le passage des artistes country-western de la radio au disque ainsi que l'émergence de nouveaux styles dans l'industrie du disque. De plus, la compagnie canadienne Compo et son étiquette Starr ne semblent pas avoir été trop affectées par la grève des musiciens, ayant fait paraître, selon

\footnotetext{
${ }^{7}$ Ibid., p. 89.

98 Thérien, L’histoire de l'enregistrement sonore au Québec et dans le monde, p. 199.

99 Ibid., p. 204.
} 
l'état de la collection de Bibliothèque et Archives nationales du Québec (BAnQ) pour ces années, au moins 160 disques, peut-être en raison de la forte proportion de musiciens non affiliés dans leur écurie $^{100}$. Chez RCA Victor cependant, selon Jean-Jacques Schira et Robert Giroux, la grève interrompt tout enregistrement ${ }^{101}$. C'est peut-être pour cette raison que la compagnie a attendu trois ans avant de répliquer à Starr en produisant son premier chanteur countrywestern, Paul Brunelle, dont les deux premiers disques paraissent en $1945^{102}$.

Les enregistrements sonores produits par les artistes countrywestern présentent un ensemble de caractéristiques relativement homogènes. Les interprètes font un usage abondant et structuré de traits vocaux rattachés à l'expressivité country, dans une version légèrement adaptée, mais employée selon des règles très strictes ${ }^{103}$. L'univers western et la nature sont très présents dans les paroles des chansons. Une certaine nostalgie est mise en valeur, notamment dans le traitement des récits sentimentaux ${ }^{104}$. De leurs débuts à la radio, où ils sont souvent interprètes de demandes spéciales, et de leurs tournées provinciales, où ils se font les diffuseurs des grands succès

100 Comme le souligne Robert Thérien, la grève des musiciens explique peut-être également pourquoi Lionel Parent a adopté des pseudonymes sur disque entre 1942 et 1944 (Robert Thérien, "Lionel Parent, auteur, compositeur et interprète (1905-1980) ", Le Gramophone virtuel, sur le site de Bibliothèque et Archives Canada, 2010, [http://www.lac-bac.gc.ca/gramophone/028011-1064-f.html] [15 mai 2016]).

101 Jean-Jacques Schira et Robert Giroux, "La discographie : un instrument de mesure de la popularité (au Québec 1898-1959) », dans Robert Giroux (dir.), La chanson dans tous ses états, Montréal, Tryptique, 1987, p. 58.

102 Mario Gendron ("Paul Brunelle », Portraits de la culture montérégienne) indique, en effet, que les quatre premières chansons enregistrées par Paul Brunelle, parues sur ses deux premiers disques, ont été enregistrés en 1944. Selon Robert Thérien («Lionel Parent, auteur, compositeur et interprète (1905-1980) »), ces deux disques ont paru en octobre et en novembre 1945.

103 Voir Catherine Lefrançois, La chanson country-western, 1942-1957: un faisceau de la modernité culturelle au Québec, thèse de doctorat, Québec, Université Laval, 2011.

${ }^{104}$ Lefrançois, "Le train qui siffle" ". 
de la musique populaire de l'heure, les artistes country-western conservent, même sur disque, des styles individuels distinctifs. Les pionniers du country-western interprètent chacun un répertoire typique, et leurs styles vocaux ainsi que leurs performances scéniques sont tout aussi personnels. Willie Lamothe chante sur scène des chansons fantaisistes inspirées de ses idoles Maurice Chevalier et Charles Trenet, et ses tournées incluent de la comédie et même des artistes forains comme Michel Messier ${ }^{105}$. Il est connu, sur disque, pour ses chansons joyeuses, son style exubérant et l'usage abondant du yodel. Paul Brunelle adopte lui aussi sur scène le modèle du spectacle de variétés avec la formule de la soirée du bon vieux temps. Chanteur sérieux, il possède une maitrise vocale exceptionnelle et son yodel est souvent virtuose. Marcel Martel, un émule de Roland Lebrun et de Tino Rossi dont, dit-il, il faisait " les imitations les plus authentiques $^{106}$ ", est, quant à lui, le spécialiste de la chanson sentimentale et plaintive, une marque de commerce qui perdure : en 1965, d'après Gérald Godin, il est " celui qui plaît le plus aux femmes ${ }^{107}$ ".

Entre 1942 et 1958, les chanteurs country-western enregistrent pour Compo et RCA Victor, les deux seules compagnies de disques canadiennes à avoir survécu à la Crise. Jusqu'à la fin des années 1950, le country-western ne possède aucune institution autonome par rapport au reste de la musique populaire. La structuration s'amorce doucement avec l'arrivée, au cours des années 1950, de nouvelles maisons de disques qui s'intéressent au country-western et qui produisent exclusivement de la musique populaire. En 1952, le disquaire Rosaire Archambault fonde la compagnie Alouette, qui offre des microsillons à prix compétitifs et de styles musicaux variés ${ }^{108}$.

\footnotetext{
105 Le Serge, Willie Lamothe, p. 69.

106 Martel et Boulanger, Au jardin de mes souvenirs, p. 29.

107 Godin, «Ils ont inventé le cowboy québécois », p. 41.

108 Cécile Huot, "Archambault Musique ", dans Encyclopédie canadienne, dernière modification : 15 décembre 2013, [En ligne], [http://www.encyclopediecanadienne. ca/fr/article/archambault-musique/] (17 mai 2016).
} 
L'entreprise fait paraître des enregistrements de Maurice Bienvenue. Pour la première fois, un chanteur country-western est présenté comme tel par une maison de disques, et Maurice Bienvenue enregistre sous le nom de Jimmy «Le cowboy » Debate ${ }^{109}$. En 1958, la compagnie Rusticana, fondée par Roger Miron ${ }^{110}$, chanteur country-western qui avait connu un grand succès en 1956 avec la chanson $\grave{A}$ qui l'ptit cour après neuf heures ${ }^{111}$, fait paraître son premier disque. Une bonne partie de son catalogue est consacrée à la chanson country-western, mais d'autres styles musicaux associés à la danse y figurent aussi. Parmi les premiers disques produits par Rusticana, on compte les enregistrements de Léo Benoît, qui allie country et rock and roll ${ }^{112}$.

Au terme de cette longue période de structuration, le countrywestern possède un embryon d'industrie spécialisée, et ses pionniers ayant connu le plus de succès sont désormais des musiciens professionnels à temps plein. Des compagnies généralistes vont commencer, dès la fin des années 1950, à mettre en marché des rééditions d'enregistrements country-western à succès (Carnaval), puis MCA

109 L'orthographe de ce pseudonyme varie d'un enregistrement à l'autre ainsi que d'une source à l'autre (Dabate, D'Abate, Debate). L'orthographe Debate semble être la plus répandue.

110 Musée du rock’n'roll du Québec, "Roger Miron ", Panthéon du rock’n'roll Québécois, sur le site du Musée du rock'n'roll du Québec, [http://museedurocknroll.com/ pantheon-du-rocknroll-quebecois/] (20 août 2011).

111 Des indices du succès de cette chanson se trouvent dans l'autobiographie de Marcel Martel. Roger Miron enregistrait pour RCA Victor et Marcel Martel reprenait pour Starr À qui l'ptit cœur après neufheures, une pratique qui était alors courante. Marcel Martel, qui semble s'approprier la paternité de cette chanson dans ses souvenirs, raconte : "Côté disque, j'ai fait un genre de malheur à la fin de mars-début avril en sortant "À qui l'petit cour après neuf heures" [sic]. Ce disque a tourné un peu partout au Québec. Certains annonceurs de radio le présentaient pour rire de moi, d'autres y trouvaient du plaisir, mais la chanson ne laissait personne indifférent. La compagnie Apex avait du mal à satisfaire la demande. [...] Dans les boîtes à musique, le disque tournait des dizaines et des dizaines de fois par jour. Même dans les villages les plus éloignés, on a dû changer quelquefois la copie dans les "guiboux" [juke-box], tellement elle était usée " (Martel et Boulanger, Au jardin de mes souvenirs, p. 146).

112 Rusticana et sa filiale Click feront aussi paraître de nombreux disques de rock and roll et de yé-yé. 
Coral, qui offrent des réimpressions de certains enregistrements originaux de l'étiquette Starr sur 33 tours ${ }^{113}$. La compagnie London, filiale canadienne de la maison mère britannique Decca ${ }^{114}$, fait paraître, pendant les années 1960, des albums longue durée de Marcel Martel, de Willie Lamothe et de Paul Brunelle. Pour London, ces trois pionniers du country-western semblent très rentables. En 1965, un représentant de la compagnie confie : "On ne les échangerait pas pour n'importe qui dans la chanson à l'heure actuelle. Ils sont des valeurs sûres. Lamothe vend 30000 copies de chaque microsillon qu'il sort. Brunelle en vend entre 40000 et 45000 . Martel entre 15000 et $16000^{115}$."

\section{Conclusion}

La chanson country-western émergente s'est intégrée à des réseaux bien établis. Celui des stations de radio, dont il a surtout été question ici, lui a offert un lien privilégié avec l'auditoire et un tremplin vers les studios d'enregistrement montréalais. D'autres auraient pu être évoqués. Celui des troupes de variétés, tout comme la radio, a permis à plusieurs artistes amateurs de se professionnaliser. Celui des musiciens de folklore a fourni aux chanteurs country-western à la fois une banque de musiciens accompagnateurs et un circuit de tournées déjà constitué. Le genre country-western s'est donc développé en partie en continuité avec les grands pôles de la vie culturelle des années 1940, dont les institutions, les modes de fonctionnement et les organes de diffusion lui ont permis de se structurer non pas en marge mais au sein même de l'industrie musicale. L'émergence du country-western, entre 1942 et 1958, s'est opérée dans des conditions industrielles et économiques très

\footnotetext{
113 Yves Claudé (sous le pseudonyme d'Yves Alix), «L'industrie du western », Chansons d'aujourd'hui, vol. 9, no 2 (mars-avril 1986), p. 21.

114 Edward B. Moogk, "London Records of Canada Ltd. ", dans Encyclopédie canadienne, dernière modification : 16 décembre 2013, [En ligne], [http://www. encyclopediecanadienne.ca/fr/article/london-records-of-canada-ltd/] (mai 2016).

115 Godin, "Ils ont inventé le cowboy québécois », p. 25.
} 
favorables. La pénurie de disques français et états-uniens qui touche les deux compagnies de disques établies à Montréal, les généralistes Compo et RCA Victor, ouvre une brèche favorable à l'arrivée de nouveaux styles et de nouveaux artistes. Les besoins grandissants de la radio, en pleine expansion, permettent à des débutants et à des amateurs d'avoir facilement accès aux ondes. À cette époque, le country-western est pleinement intégré aux médias de masse et à une industrie musicale tournée vers le grand public, et il génère des produits de grande consommation dont la mise en marché s'apparente à celle des autres disques de musique populaire.

La marginalité souvent évoquée du country-western a une origine tardive. Elle a été causée par la crise du disque des années 1980, qui, ayant provoqué la fermeture des principales compagnies de disques country-western, a poussé plusieurs artistes à se tourner vers l'autoproduction. Elle correspond à un troisième temps de son histoire, lequel succède à une période de grande rentabilité, probablement stimulée par l'énorme succès commercial rencontré par des pionniers désormais au faîte de leur carrière. Il aura fallu attendre le début des années 1970 pour qu’apparaisse une compagnie s'intéressant exclusivement à ce qu'on désignait alors sous le nom de " musique campagnarde ». Fondée en 1971 ou en $1972^{116}$ par Jean Chaput, la maison de disques Bonanza, située à Ville Saint-Laurent ${ }^{117}$, propose, en 1986, un catalogue d'environ 600 microsillons ${ }^{118}$ de country-western et de musique folklorique. Les années 1970 voient naître une véritable industrie spécialisée de la musique country-western, qui connaît une période florissante avec la création de nombreuses étiquettes. Au

116 En 1986, Jean Chaput raconte à Yves Claudé (voir la note 113) avoir fondé, dix-sept ans plus tôt, la compagnie Budget, puis trois ans plus tard, Bonanza, ce qui ferait remonter les débuts de cette compagnie à 1972; le plus ancien disque produit par Bonanza conservé à Bibliothèque et Archives nationales du Québec (BAnQ) est cependant daté, selon l'institution, de 1971 (On s'en vient vite, de Lorraine Arseneault Diotte).

117 Selon les données de catalogue de BAnQ.

118 Claudé, "L’industrie du western ", p. 21. 
cours de la décennie, le chanteur Gaétan Richard crée les étiquettes Ouesteurne, Guitare et Paysanne ${ }^{119}$. Les disques Amical, créés à la même époque, produisent eux aussi du folklore et du country-western. Cependant, c'est Bonanza qui semble dominer le marché avec son imposant catalogue et des activités se déployant à la radio et à la scène. Bonanza semble avoir entretenu des liens étroits avec la station de radio CKVL, avec qui la compagnie organise un « Festival western à la salle du Plateau (Bonanza-CKVL) " à Montréal en $1979^{120}$.

Dans les années 1970, les artistes country sont représentés par l'Association de musique folklorique et campagnarde du Québec qui décerne des prix lors du festival de Saint-Pie-de-Bagot ${ }^{121}$-, dirigée par Roger Charlebois, rédacteur de la chronique " Le ranch des vedettes", publiée dans le Journal des vedettes. Le mythe countrywestern est consommé : c'est une musique campagnarde. Mais c'est une musique campagnarde qui possède ses institutions montréalaises: festivals, bars (Au pied du quai, le Casino gaspésien), stations de radio spécialisées. Et c'est aussi une musique campagnarde qui fonctionne à l'intérieur du système de vedettariat : de 1970 à 1975, Télé-Métropole présente l'émission Le Ranch à Willie, qui attire plus de 1400000 téléspectateurs par semaine ${ }^{122}$. Ces caractéristiques sont déjà présentes dans le modèle proposé dans les années 1940 par les pionniers de la chanson country-western, alors que pour la première fois, un groupe de chanteurs amateurs, d'origine ouvrière, issus de petits milieux urbains, investissent la radio et le disque pour rencontrer la faveur d'un public nombreux et chanter, en ville, la vie à la campagne.

\footnotetext{
119 Ibid.

${ }^{120}$ Yves Claudé (sous le pseudonyme d'Yves Alix), « Le western, une musique qui vient du cœur ", Mouvements, vol. 4, nº 2 (avril-mai-juin 1986), p. 50.

121 Martel et Boulanger, Au jardin de mes souvenirs, p. 336.

122 Chamberland, "La chevauchée "lyrique” de la musique western », p. 211.
} 